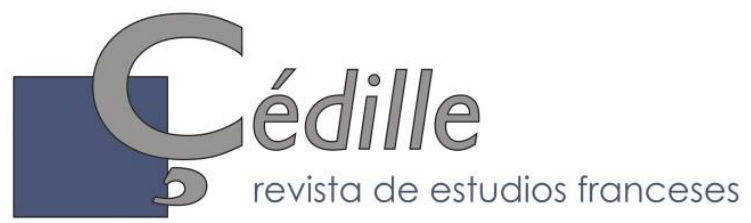

ISSN: $1699-4949$

$n^{0} 18$ (otoño de 2020)

Monografías 11

Epistemocrítica: análisis literario y saber científico

Amelia Gamoneda Lanza \& Francisco González Fernández, editores científicos

\title{
Pour une archéologie des usages savants du littéraire : remarques sur les présupposés d'un literary turn
}

\author{
Anne-Gaëlle WEBER \\ Université d'Artois \\ agaelle.weber@univ-artois.fr \\ ORCID: 0000-0002-0424-0904
}

\section{Resumen}

Para exponer sus trabajos, numerosos especialistas en humanidades, artes y letras se sirven ahora de formas literarias, desde el ensayo a la novela. La historia de los usos que los estudiosos han hecho de lo «literario», a medida que las disciplinas se han desarrollado en los siglos XVIII y XIX, puede permitir calibrar las consecuencias que tienen hoy en día en las diferentes formas de ciencia y literatura.

Palabras clave : Literatura, Disciplina, Usos, Adorno, Documento, Heurística.

\section{Résumé}

De nombreux spécialistes de sciences humaines, d'arts et de lettres usent désormais pour exposer leurs travaux de formes littéraires, allant de l'essai au roman. L'histoire des usages qu'ont pu faire les savants du « littéraire », au fur et à mesure de la constitution des disciplines aux $\mathrm{XVIII}^{\mathrm{e}}$ et $\mathrm{XIX}^{\mathrm{e}}$ siècles, peut permettre de mesurer les conséquences qu'ils ont de nos jours sur différentes formes de sciences et de littérature. Mots clés : Littérature, Discipline, Usages, Ornement, Document, Heuristique.

\begin{abstract}
Many scholars in the social sciences, arts and humanities now exhibit their works by using literary forms, ranging from essays to novels. The history of the uses that scholars have given to literature, as disciplines have developed in the 18th and 19th centuries, allows us to assess the consequences that nowadays they have on different forms of sciences and literature.
\end{abstract}

Keywords : Literature, Discipline, Uses, Adornement, Document, Heuristics.

\section{Introduction}

L'usage de formes littéraires et artistiques pour exposer et diffuser des travaux savants s'est considérablement répandu ces derniers temps dans le domaine

* Artículo recibido el 25/02/2020, aceptado el 29/10/2020. 
des recherches en arts, lettres et sciences humaines et sociales, au point que l'on pourrait parler d'un literary turn ${ }^{1}$. Sans doute est-ce là la conséquence de la double injonction politique qui pèse sur les chercheurs en sciences : celle d'abolir plus ou moins les frontières disciplinaires et celle de démontrer le « rayonnement » de leurs travaux lorsqu'ils ne peuvent se targuer d'applications techniques, sociales ou économiques immédiates.

L'essai et le roman de l'historien Ivan Jablonka - L'Histoire est une littérature contemporaine. Manifeste pour les sciences sociales (2014) et Laëtitia ou la fin des hommes (2016) - ont récemment constitué le symptôme le plus visible et le plus fameux de cet hypothétique «tournant $»^{2}$. L'usage par l'historien de la forme ou de l'écriture littéraire est doublement réflexif ; non seulement il entraîne une critique des présupposés de sa propre discipline et l'invention en acte de nouveaux critères de scientificité mais il engage une représentation de la littérature ou du littéraire qui n'est pas sans conséquence sur la manière dont on entend les définir, que l'on soit d'ailleurs chercheur en Histoire ou en littérature. Ajoutons qu'il est fort probable que ce que l'historien nomme « littérature » réponde aux critères qui conviennent le mieux aux présupposés de sa propre discipline.

Dans L'Invention du quotidien, Michel de Certeau (1980) montre qu'envisager le «quotidien » du point de vue de l'usage de ses objets revient à analyser les pratiques des usagers et la tournure de ce qui se prête à l'usage. Et si le mot d'usage semble généralement réservé aux objets techniques, Thomas Benatouill rappelle qu'étendre l'application du terme d' ' usage » à des textes ou des discours revient à admettre « la possibilité d'un rapport pragmatique et technique à des textes et des concepts » (Benatouïl, 2014 :31). Évoquer l'usage du « littéraire » supposerait donc, en première approche, de considérer que le « littéraire » soit un répertoire de techniques. Cela n'est pas inconcevable si l'on se souvient des liens essentiels qui ont existé entre la rhétorique, la poétique et la littérature et qui ont en partie subsisté par delà l'émergence de la littérature comme science. Mais en choisissant volontairement de ne pas définir a priori la littérature et de nous en tenir à l'observation de qualités prêtées à ce qui semble en relever, nous postulerons que les « usages » du littéraire dans le domaine des sciences, à partir du moment où ils contribuaient à définir ces « sciences », ont invariablement entraîné des réflexions sur la définition des outils usités et, particulièrement, des considérations sur la possibilité que les formes littéraires empruntées puissent non seulement influencer le fond savant mais participer même à son élaboration. En d'autres termes, il s'agira moins pour nous d'observer les transpositions de techniques, de textes ou de formes littéraires dans le domaine établi des disciplines

\footnotetext{
${ }^{1}$ Sur le modèle du linguistic turn des années 1970 où des historiens, des sociologues et des ethnologues promurent le rôle décisif du langage dans la construction de réalités sociales.

${ }^{2}$ Mais on pourrait également citer les travaux des géographes et, notamment, de Marc Brosseau ( $c f$. à ce sujet Marc Brosseau et Micheline Cambron, 2003 : 525-547), ceux des sociologues et notamment l'ouvrage d'Anne Barrère et Danilo Martucelli (2009), ceux de l'anthropologue Albert Piette (2017) et de ses expériences autour d'une " phénoménographie existentiale » et, plus généralement, nous soulignerons le développement sans précédent des formes essayistiques, voire du recours à la bande dessinée.
} 
scientifiques que de nous situer du point de vue de l'usage pour guetter l'émergence conjointe de critères de scientificité et de littérarité ${ }^{3}$.

Or, si l'élaboration contemporaine de nouveaux critères de scientificité et la nécessité apparente d'abolir les frontières disciplinaires passent par des usages de formes et d'écritures artistiques, l'émergence de disciplines savantes et la spécialisation des sciences, au tournant des $\mathrm{XVIII}^{\mathrm{e}}$ et $\mathrm{XIX}^{\mathrm{e}}$ siècles, ont précisément entraîné, de la part des tenants de ces nouvelles «sciences » des usages parfois polémiques de ce qui se constituait alors en « littérature ». Tout se passe comme si la « littérature » ou, du moins, comme si une certaine conception de la « littérature » en tant que méthode et corpus devait constituer systématiquement la pierre de touche de la naissance de nouvelles «sciences » et de l'organisation, entre elles, de ces savoirs. Ce parallèle, aussi paradoxal semble-t-il, traduit le malaise contemporain des sciences humaines et sociales, en quête d'une légitimité et d'une scientificité qu'en leur temps l'astronomie (ou un certain devenir de l'astronomie), la géologie, la physiologie, la médecine ont dû revendiquer. En situant cependant le point de départ de nos analyses des usages du «littéraire » par les tenants de disciplines qui inventent des méthodes, des objets et des corpus, nous entendons saisir précisément les moments où les usages de ce qui est en passe de devenir radicalement étranger (les «lettres ») sont explicités et justifiés par les savants et, donc, en quelque sorte, «fondés ». Les définitions de la « littérature » découleront de ses usages que nous classerons a priori en usages ornementaux, documentaires et heuristiques, suivant une logique qui va de l'idée de l'usage comme recours à une technique jusqu'à l'usage comme facteur de transformation de l'outil et de l'objet.

\section{L'usage ornemental}

L'usage ornemental du « littéraire » est celui qui use de formes rhétoriques ou poétiques pour séduire un large public et orner le discours savant ; il n'est pas neuf au XVIII ${ }^{\mathrm{e}}$ siècle et est parfaitement compatible avec l'idée d'une science destinée à un large public et celle d'unité des savoirs et de leur objet. Hugues Marchal souligne dans Muses et Ptérodactyles (Marchal, 2013 : 9-13) que le continent oublié de la poésie dite scientifique ou didactique a longtemps incarné le présupposé encyclopédique suivant lequel la poésie pouvait être poésie et diffuser les savoirs de son temps. Le développement du dialogue scientifique reposait sur le même présupposé (Chassot, 2011: 16-19). Or la spécialisation d'un grand nombre de disciplines savantes au tournant des $\mathrm{XVIII}^{\mathrm{e}}$ et $\mathrm{XIX}^{\mathrm{e}}$ siècles passe justement par l'invention d'un langage qui s'éloigne du vocabulaire commun, par la définition d'objets propres et par une professionnalisation des sciences. L'usage de formes littéraires semble alors une «traduction» du vocabulaire savant qui

\footnotetext{
${ }^{3}$ Ce travail s'inscrit donc dans la lignée de l'anthologie Littérature et sciences au XIX ${ }^{e}$ siècle dirigée par Nicolas Wanlin (2019) qui recense et commente les définitions croisées du scientifique et du littéraire et démontre par l'exemple la profonde fécondité du dialogue entre des sphères supposées séparées.
} 
peut s'avérer impropre à rendre compte de sa spécificité et court le risque soit de le simplifier outrageusement, soit de le déformer.

Parmi les «outils » et techniques littéraires auxquels le savant peut avoir recours figurent non seulement les genres mais aussi le style et ses figures. Car si la littérature naît en se distinguant de la rhétorique, il n'en demeure pas moins que les manuels de rhétorique et de littérature parus à la fin du XVIII ${ }^{\mathrm{e}}$ siècle constituent dans la première moitié du $\mathrm{XIX}^{\mathrm{e}}$ siècle des ouvrages de référence, comme en témoigne par exemple le Dictionnaire de littérature (1770) d'Antoine Sabatier de Castres qui n'est qu'un dictionnaire des figures de construction et de style. En 1853, Bernard Jullien publie encore un Traité de rhétorique et de littérature. Or tous ces traités ont en commun à la fois de proposer des classifications des différents styles et d'avertir les lecteurs des «abus » de l'usage des « ornements du style » (Jullien, 1866 : iii). Le style est le plus souvent défini par référence au discours sur le style prononcé lors de sa réception à l'Académie française par Buffon (1753), comme l'ordre et le mouvement qu'on met dans les pensées (Buffon, 1827 : 5), et le maître-mot de son bon usage est celui de la convenance de la diction avec le sujet. Mais ni Buffon, ni les encyclopédistes, ni Voltaire dans le Dictionnaire philosophique ne condamnent à proprement parler le recours aux « ornements » du style, à condition qu'on en use avec précaution. Ainsi le Chevalier de Jaucourt qui rédige l'article "Style » de l'Encyclopédie écrit: "Quoique la beauté du style dépende des ornements dont on se sert pour l'embellir, il faut les ménager avec adresse ; car un Style trop orné devient insipide ; il faut placer la parure de même qu'on place les perles et les diamants sur une robe que l'on veut enrichir avec goût»(Encyclopédie, 1786 : 16). Et le style «fleuri » est très tôt érigé en catégorie propre «qui admet toutes les figures et tous les ornements du discours, connus sous le nom de fleurs d'éloquence » (Castres, $1770: 495$ ), sans qu'on en use de manière péjorative.

La spécialisation grandissante des disciplines savantes, si elle rend nécessaire le fait de justifier de l'usage du « style », ne signifie pas nécessairement que les savants y renoncent. D'autant que certains d'entre eux savent que le style d'un écrit peut concourir à sa légitimation savante aux yeux du public instruit comme à ceux des savants. En d'autres termes, si le style, en tant qu'ordre du discours, est une attente de tout texte savant, le style en tant qu'ornement et que figures est un mal nécessaire.

John Playfair (1748-1819), professeur de mathématique et de physique à l'université d'Édimbourg, a ainsi entrepris en 1802 de réécrire les articles savants de James Hutton et d'exposer l'hypothèse d'une origine volcanique du monde dans Illustrations of the Huttonian Theory of the Earth afin de donner à cette théorie l'audience dont l'expression obscure et contournée de son inventeur l'a privée. C'est du moins ce que rappelle son fils James Playfair dans les mémoires biographiques qu'il consacre à son père : "The peculiar style of composition and arrangement adopted by Dr. Hutton, both in the sketch published in the Transactions of the Royal Society of Edinburgh and in the more extended work which followed, rended this theory less intelligible, and much less known that its merits deserved». La récriture proposée par son père est au contraire universellement reconnue pour 
constituer un modèle de "pureté de la diction" ("purity of diction»), de simplicité du style (« simplicity of style ») et de clarté des explications («clearness of explanation ») (Playfair, 1822 : xix-xx). Il importe donc à John Playfair, comme à son fils, que l'usage du style en science s'éloigne de l'idée d'une pure ornementation, même si l'un et l'autre sacrifient sans le dire à l'idéal horacien du plaire et de l'instruire.

John Playfair fait en effet référence à l'Ars poetica en 1812 lorsqu'il commente la réédition des Elements of Geometry par John Leslie en insistant sur l'élégance reconnue de l'écriture d'Euclide :

It should also be considered, that the language of Geometry requires nothing but accuracy and perpicuity, united to as much conciseness as is consistent with the latter. The works in that science, by the adaptation of their parts, may exemplify quantum series, juncturaque pollet; but can hardly illustrate any other of the rules of the critic of the orator. They admit no expression purely ornamental and reject thing that can withdraw the attention from the main object. To metaphor and variety of expression they are particulartly averse; and the geometer must never forget, that the transparency of a medium may be injured by the flowers scattered on its surface, no less than by the mud diffused through its mass (Playfair, 1812:97) ${ }^{4}$.

La citation empruntée à Horace est volontairement tronquée ; le poète écrivait : "Tantum series juncturaque pollet tantum de medio sumptis accedit honoris » (Horace, 1821 :462). Horace alors traitait du poème dramatique et suggérait qu'un imitateur qui croirait faire une œuvre en empruntant son sujet à un autre et en utilisant le vocabulaire commun se tromperait, tant la qualité littéraire de l'œuvre et sa singularité reposent sur l'ordre et sur l'éclat qu'on sait donner au vocabulaire commun. Or Playfair réduit volontairement le précepte horacien à l'idée de composition et semple rejeter, comme particulièrement peu conformes au fond géométrique les figures et les «fleurs » qu'Horace ne condamnait pas. L'écriture « littéraire » constitue donc un instrument de diffusion et de légitimation des théories savantes, à condition néanmoins qu'elle ne l'emporte pas sur le fonds et parvienne à concilier le plaisir de la lecture avec le sérieux de l'objet traité.

\footnotetext{
${ }^{4}$ « Il faut aussi tenir compte du fait que le langage de la géométrie n'exige rien d'autre que la précision et la perspicacité, unies à autant de concision qu'il est cohérent avec cette dernière. Les œuvres de cette science, par l'adaptation de leurs parties, peuvent illustrer quantum series, juncturaque pollet; mais ne peuvent guère illustrer d'autres règles de la critique de l'orateur. Ils n'admettent aucune expression purement ornementale et rejettent des choses qui peuvent détourner l'attention de l'objet principal. À la métaphore et à la variété d'expression, ils sont particulièrement opposés ; et le géomètre ne doit jamais oublier que la transparence d'un médium peut être blessée par les fleurs éparpillées sur sa surface, pas moins que par la boue qui se diffuse à travers sa masse». Nous traduisons.
} 
Georges Cuvier, successeur de Buffon à la tête du Muséum, revient lui aussi, au long des éloges historiques qu'il livre en tant que secrétaire perpétuel de l'Académie des sciences, sur l'usage nécessaire, mais parfois abusif, du « beau » style et sait fort bien en explorer les avantages et les limites. Il discrédite notamment ses pairs en les qualifiant d'écrivains usant, pour leur seul éclat, des ornements du style. Il suggère ainsi, au cœur de l'éloge historique de Daubenton, que Buffon, s'il ne l'avait pas rencontré, aurait pu n'être qu'un «écrivain »: «Mais si la vérité n'avait pas fait la base de son travail, s'il avait prodigué les brillantes couleurs de sa palette à des dessins incorrects ou infidèles, s'il n'avait combiné que des faits imaginaires, il aurait bien pu devenir un écrivain élégant, un poète ingénieux ; mais il n'aurait pas été un naturaliste, il n'aurait pu aspirer au rôle qu'il ambitionnait de réformateur de la science »(Cuvier, 1861b:6). L'éloge est ambigu ; il dessine les contours d'un peintre « à la palette brillante » susceptible d'exercer son talent de coloriste sur n'importe quel objet et incapable de se livrer seul au travail patient de l'étude savante.

Mais Cuvier ne s'arrête pas là. Il informe l'éloge en un véritable morceau de bravoure littéraire, dressant un parallèle entre Daubenton et Buffon : En effet on peut dire que jamais association ne fut mieux assortie. Il existait au physique et au moral, entre les deux amis, ce contraste parfait qu'un de nos plus aimables écrivains assure être nécessaire pour rendre une union durable ; et chacun d'eux semblait avoir reçu les qualités propres à tempérer celle de l'autre par leur opposition.

Buffon, d'une taille vigoureuse, d'un aspect imposant, d'un naturel impérieux, avide en tout d'une jouissance prompte, semblait vouloir deviner la vérité, et non l'observer. Son imagination venait à chaque instant se placer entre la nature et lui, son éloquence s'exercer contre sa raison avant de s'employer à entraîner celle des autres.

Daubenton, d'un tempérament faible, d'un regard doux, d'une modération qu'il devait à la nature autant qu'à sa propre sagesse, portait dans toutes ses recherches la circonspection la plus scrupuleuse; il ne croyait, il n'affirmait que ce qu'il avait vu et touché, bien éloigné de vouloir persuader par d'autres moyens que l'évidence même, il écartait avec soin de ses discours ou de ses écrits toute image, toute expression propre à séduire [...] (Cuvier, 1861b :7).

La forme canonique du portrait croisé constitue en acte l'« union » des contraires dont il est question. Cuvier souligne d'ailleurs son emprunt à la littérature en évoquant « un nos plus aimables écrivains », c'est-à-dire probablement qui pourrait fort bien être Jaques Henri Bernardin de Saint-Pierre pour qui les « harmonies » président aux lois naturelles et humaines et dont les deux célèbres héros, Paul et Virginie, sont précisément décrits en 1788 sous la forme du portrait croisé (Bernardin de Saint-Pierre, 1984 : 122-123). En acte donc, et au détour d'un éloge destiné à un large public, Cuvier à la fois condamne un usage du style et du discours littéraire qui séduit davantage qu'il ne persuade et use d'une forme littéraire 
qui convainc son lecteur de la nécessité de recourir en sciences avant toute chose à l'observation et de ne livrer ses résultats qu'à partir d'une forme qui puisse coïncider parfaitement avec le fond, sans l'obscurcir ou l'alourdir de vains ornements.

Mais la condamnation d'un certain style et de son usage n'est pas seulement liée à la volonté d'influencer la réception des œuvres savantes par le grand public ; ils touchent plus fondamentalement aux présupposés et à la définition de la visée et de la vérité de l'histoire naturelle.

Lorsqu'en 1827, Georges Cuvier écrit l'éloge de Lacépède, disciple de Buffon et auteur d'une Histoire naturelle des poissons, le savant dénonce cette fois la confusion entretenue par Buffon entre la logique des tropes et celle des lois naturelles : «Chacun voit que ce n'est qu'en se faisant illusion par l'emploi d'un langage figuré qu'il a pu attribuer à des molécules organiques la formation de cristaux ». Lacépède, lui, n'a aucune excuse puisqu'il a osé, en physique, se passer du calcul et de l'expérimentation (Cuvier, 1861b : 382).

Derrière le constat de l'impossibilité d'user du langage figuré dans des disciplines savantes où le langage mathématique a pris le pas sur le langage commun et où l'observation et l'expérimentation sont possibles, se fait jour l'opposition entre les savants qui tiennent encore pour une unité des savoirs et, surtout, pour une unité de la Nature et ceux qui défendent le caractère "positif » des sciences.

Le débat qui opposa à l'Académie en 1830 Georges Cuvier et Étienne Geoffroy Saint-Hilaire sur l'« unité de composition » l'illustre parfaitement. De cette querelle, Geoffroy Saint-Hilaire rend compte dans Principes de philosophie zoologique en 1830. Dès le discours préliminaire, l'auteur s'interroge sur les résistances qu'opposent des savants aux théories et aux innovations et se demande s'il faut conserver une ancienne méthode pour la détermination des organes « en reconnaissance de ses anciens et utiles services ", se posant d'emblée en l'inventeur à la fois de nouvelles méthodes, de nouvelles idées et de nouvelles manières de définir l'histoire naturelle (Geoffroy Saint-Hilaire, $1830: 2$ ). À Cuvier, qui a feint pendant les débats de se poser en chef de file des «naturalistes ordinaires », suggérant par là que les tenants de l'unité de composition et de la philosophie zoologique, relevaient d'une science extraordinaire, pour ne pas dire extravagante, Geoffroy Saint-Hilaire répond en revendiquant son titre de savant et de savant « ordinaire »: « Je me renferme dans le devoir de la plus stricte observation des faits ; je ne prétends qu'au rôle d'historien de ce qui est » (Geoffroy Saint-Hilaire, 1830 : 16). Établir des « rapports philosophiques » à partir de ressemblances ou d'analogies observées entre les espèces n'est pas selon Geoffroy Saint-Hilaire la même chose que de présupposer une causalité entre ces analogies et donc s'engager sur la voie du vraisemblable et de la théologie.

Or en 1829, Geoffroy Saint-Hilaire avait déjà défendu l'unité de composition dans l'article «Nature » de l'Encyclopédie moderne qui nuançait l'article « Nature » que Cuvier avait composé pour le Dictionnaire d'histoire naturelle en 1822. Déjà Cuvier avait reproché à son confrère de céder là au panthéïsme : «Ces vues d'unité sont renouvelées d'une vieille erreur née au sein du panthéïsme, étant 
principalement enfantée par une idée de causalité, par la supposition inadmissible que tous les êtres sont créés en vue les uns des autres » (Geoffroy Saint-Hilaire, 1820 : 59). Mais Cuvier tient à l'idée que les êtres ont un en soi, ce que Geoffroy Saint-Hilaire ne dément pas lorsqu'il cite la remarque de Cuvier en note des Principes de philosophie zoologique et répète les objections qu'il avait lui-même formulées en distinguant l'établissement scientifique de rapports philosophiques et généraux entre les faits observés et la sujétion à l'idée de causalité. La note de Geoffroy Saint-Hilaire surgit au moment où il retrace l'un des discours que Cuvier a prononcés pour lui répondre à l'Académie et, plus précisément encore, au moment où Cuvier accuse son confrère d'user de manière métaphorique de termes tels que «plan » ou « composition » qu'il entend définir lui-même par une comparaison avec l'architecture, empruntée de facto à l'article «Nature » de l'Encyclopédie.

Car d'emblée, la réponse de Cuvier s'est ouverte par une longue et brillante péroraison où il entend faire la part des choses entre le discours savant et l'usage abusif des tropes :

Dans toute discussion scientifique, la première chose à faire est de bien définir les expressions que l'on emploie ; sans cette précaution l'esprit s'égare promptement; prenant les mêmes mots dans un sens, à un endroit du raisonnement, et dans un sens différent à un autre endroit, on fait ce que les logiciens appellent un syllogisme à quatre termes qui sont les plus trompeurs des sophismes. Que si, dans l'exposé de ces mêmes raisonnements, au lieu du langage simple des mots propres, rigoureusement exigés dans les sciences, on emploie des métaphores et des figures de rhétorique, le danger est bien plus grand encore. On croit se tirer d'embarras par un trope, répondre à une objection par une paronomase, et en se détournant ainsi de sa route directe, on s'enfonce promptement dans un labyrinthe sans issue (Geoffroy SaintHilaire, $1830: 58$ ).

L'opposition entre Geoffroy Saint-Hilaire et Cuvier n'est pas une simple querelle de mots : l'usage de certaines figures de style ou métaphores nécessaires, à commencer par le simple mot de «nature », peut valoir mésusage aux yeux de ceux qui accordent au terme un sens différent. Plus fondamentalement encore, les tropes et les figures engagent la manière dont le naturaliste entend ou non découvrir les lois de la Nature, voire remonter aux causes premières et, corrélativement, signalent par l'usage qu'on en fait différentes manières de concevoir la visée du discours savant et d'apprécier les critères de sa scientificité. Car Geoffroy se plaint aussi que ses vues aient été comparées aux « visions romanesques » de Telliamed (Geoffroy Saint-Hilaire, 1830 : 67), signalant par là la confusion que font ses détracteurs entre la vérité à laquelle peuvent prétendre les généralisations philosophiques et l'invraisemblance à laquelle le « roman» est toujours réduit, du point de vue des savants. 
Le « littéraire », dans le cadre de l'usage ornemental qu'en font les savants, semble désigner à la fois un répertoire de formes et de figures et la fiction romanesque. Plus fondamentalement, il se réduit à une manière d'écrire destinée avant tout à plaire et à séduire. Mais ces acceptions recoupent des avis extrêmement nuancés. En effet, s'il semble admis qu'il faut y avoir « recours », ne serait-ce que pour plaire à un large public, les savants font la part des choses entre la conviction que peut emporter l'usage d'un « beau » style ou d'une forme adéquate et convenante et la séduction vaine qu'exercent les «fleurs de rhétorique » et les sophismes. Pour le dire vite, le « littéraire » est nécessairement caricaturé lorsqu'il vaut argument contre les méthodes ou les théories de ses pairs, sans pour autant que les savants ne renoncent à son usage, à condition toutefois d'en nuancer l'acception. Le «beau » style qui répond à un certain idéal de transparence est celui qui témoigne de l'adéquation de la forme au fond et la plupart de ceux qui s'en prennent au langage fleuri et à la réduction de la « littérature » à la rhétorique en tant que répertoire prônent l'usage de formes ou de figures littéraires qui ne dénaturent par leur objet mais participent en quelque sorte de sa description et de sa compréhension. Quitte d'ailleurs à inventer de nouvelles formes « littéraires » dont la poétique puisse coïncider avec les lois naturelles qu'il s'agit d'élaborer, comme le firent Alexander von Humboldt dans Kosmos ou Goethe dans Versuch die Metamorphosen der Pflanzen zu erklären (Weber, 2016 ; 2019a).

\section{L'usage documentaire}

Dans un certain nombre de disciplines savantes ayant au début du XIX siècle revendiqué leur scientificité, le fait même de ne pouvoir recourir ni à l'observation, ni à l'expérimentation, appelle l'invention de nouveaux régimes de la preuve scientifique et, partant, de critères de scientificité beaucoup plus complexes que ce que l'on a rétrospectivement retenu du siècle du scientisme. L'usage du littéraire joue là un rôle nouveau. Le texte littéraire peut en effet être conçu comme susceptible de constituer un document qui, moyennant interprétation, vaut preuve d'une théorie. Cela, d'ailleurs, en littérature, n'est pas nouveau; c'est le propre de la critique littéraire française de la fin du XIX ${ }^{\mathrm{e}}$ siècle, telle qu'elle s'incarne dans le quatuor formé par Sainte-Beuve, Taine, Brunetière, et Renan que d'avoir défendu la " scientificité » des études littéraires en inscrivant l'œuvre littéraire dans la masse des documents susceptibles de refléter soit l'histoire interne de la discipline, soit l'histoire individuelle, soit encore l'histoire collective. Mais il se pourrait bien qu'avant que l'usage documentaire du texte littéraire ne vienne justifier les méthodes scientifiques des études de la littérature, il ait, dans le discours savant, contribué à fonder en science certaines disciplines. La « littérature » est, dans le cas de ce second usage, entendue à la fois comme un corpus d'œuvres et comme un ensemble des méthodes formant les « études littéraires ».

Ainsi, certaines figures d'écrivains et certaines méthodes d'analyses ont concouru au développement de la médecine aliéniste dans la seconde moitié du $\mathrm{XIX}^{\mathrm{e}}$ siècle. Après que l'Italien Cesare Lombroso a par exemple diffusé le concept de dégénérescence pour décrire une certaine rétrogadation de l'espèce humaine, le médecin et journaliste austro-hongrois se livre, en 1892 dans Entartung, 
au commentaire littéraire de poèmes de Verlaine ou Mallarmé pour mettre en évidence les symptômes de la maladie de ces poètes :

In der Ausdrucksweise Verlaines fällt zweierlei auf. Einmal die häufige Wiederkehr desselben Wortes, derselben Wendung, jenes „Wiederkäuen“, „rabâchage“, das wir als Merkmal schwachsinnigen Denkens kennen gelernt aben. Fast in jedem seiner Gedichte werden einzelne Verse und Halbverse einigemale ungeändert wiederholt und statt eines Reimwortes erscheint oft einfach das nämliche Wort wieder. Wollte ich alle derartigen Stellen anführen, so müßte ich ungefähr seine sämmtlichen Gedichte abschreiben. Ich will also nur einige Proben geben, darunter mehrere in der Ursprache, damit ihre Eigenthümlichteit dem Leser voll zum Bewußtsein gelange. In Crépuscule $d u$ soir mystique erscheinen die Verse „Die Erinnerung sammt der Dämmerung“ und „Georgine, Lilie, Tulpe und Ranunkel“ ohne innere Nothwendigkeit je zweimal. Im Gedichte Promenade sentimentale verfolgt das Beiwort „blême“, „fahl“, den Dichter nach Art einer Zwangsvorstellung oder „Onomatomanie“ und er wendet es auf Wasserrosen und Wogen (fahle Wogen!) an. Nuit du Walpurgis classique beginnt: „,... Ein rhythmischer Sabbat, rhythmisch, äußerst rhythmisch“ $[\ldots]$

Die zweite Eigenthümlichkeit der Redeweise Verlaines ist das andere Merkmal des Schwachsinns: das Verknüpfen gänzlich unzusammenhängender Haupt- und Beiwörter, die einander durch eine sinnlos schweifende Ideen-Assoziation oder durch eine Klang ]ähnlichkeit rufen. Einige Beispiele haben wir schon in den bisher mitgetheilten Proben gefunden. Es war in diesen vom ,ungeheuern und zarten Mittelalter“ und vom „Brandmal, welches donnert“ die Rede (Nordau, 1892: 200-201).

\footnotetext{
5 « Deux choses frappent dans le langage de Verlaine. Premièrement, le fréquent retour du même mot, de la même tournure, ce « rabâchage » dans lequel nous avons vu un symptôme de débilité intellectuelle. Presque dans chacune de ses poésies reviennent plusieurs fois, sans changement, les mêmes vers et les mêmes hémistiches, et au lieu d'une rime reparaît tout simplement le même mot. Si je voulais citer tous les exemples de ce genre, il me faudrait transcrire à peu près toutes les poésies de l'auteur ; je me bornerai donc à quelques spécimens.

Dans le Crépuscule du soir mystique reparaît deux fois, sans nécessité organique, ce vers : "Le souvenir avec le crépuscule", et celui-ci "Dahlia, lys, tulipe et renoncule". Dans Promenade sentimentale, l'adjectif blême poursuit le poète à la façon d'une obsession ou "onomatomanie", et il l'applique aux nénuphars et aux ondes ("des ondes blêmes!'”). La Nuit du Walpurgis classique commence ainsi : "Un rythmique sabbat, rythmique, extrêmement/rythmique..." [...]. La seconde particularité du langage de Verlaine est l'autre symptôme de la débilité intellectuelle : la réunion de substantifs et d'adjectifs absolument incohérents, qui s'appellent réciproquement par des associations d'idées vaguant sans égard au sens, ou par similitude de son. Nous en avons déjà trouvé quelques exemples dans les citations précédentes. Il y a été question du "Moyen Âge énorme et délicat » et de la «brûlure...qui tonne" » (Nordau, 1894 : 221-228).
} 
Les « inventions » poétiques de Verlaine deviennent alors, sous la plume du médecin qui commente le texte, les symptômes de la débilité de l'individu et du poète, et des documents à l'appui d'une dégénérescence générale dont les poètes sont, selon lui, les précurseurs.

De la même manière, des documents antiques, qu'ils émanent de la culture égyptienne, gréco-latine, hindoue ou chinoise, font de gloses de la part de savants qui s'inspirent ainsi des travaux des philologues ou se posent en philologues. Cela est particulièrement vrai en astronomie ou en géologie, où le texte vient suppléer aux observations encore limitées, voire impossibles. Mais cela vaut aussi en chimie lorsque Marcelin Berthelot compose en 1885 Les Origines de l'alchimie et fait de cette histoire curieuse d'une pseudo-science le fer de lance de la défense de ses positions anti-atomistes.

Berthelot se livre à une longue préface où il décrit longuement la consultation qu'il a faite de papyrus et de manuscrits grecs, les traductions et travaux de paléographie auxquels il s'est livré afin de composer une histoire de la chimie qui puisse expliquer les raisons pour laquelle cette «science» est si tardive. L'ouvrage tout entier se présente comme un chef d'œuvre d'érudition ; il est assorti d'un appareil d'appendices qui comportent aussi bien des transcriptions de manuscrits grecs que des traductions inédites du pseudo-Démocrite et le savant n'hésite pas à mettre en évidence la manière dont il a éclairé l'histoire des idées en donnant leurs places aux auteurs méconnus des traités alchimiques grecs (Berthelot, 1885 : XIV). S'il feint de vouloir intéresser d'abord, en rédigeant une histoire de l'alchimie, les philosophes et les gens de lettres, Berthelot toutefois suggère à la fin de sa préface que «les opinions auxquelles les savants tendent à revenir aujourd'hui sur la constitution de la matière ne sont pas sans quelque analogie avec les vues profondes des premiers alchimistes » (Berthelot, 1885: XV). Et l'ouvrage alors s'informe en une histoire de la chimie, ou de la théorie d'une matière fondamentale défendue en chimie par Marcelin Berthelot qui, parce qu'elle a une histoire, gagne une certaine légitimité. L'exposé des anciens traités alchimiques vaut avertissement aux détracteurs de Berthelot qui défendent les théories atomistes et pourraient bien, aux yeux des futurs chimistes, passer pour aussi extravagants que les alchimistes dont il a traité (Berthelot, 1885 : 321). La glose, la traduction et l'analyse philologique de manuscrits anciens sont donc mises au service de théories jusqu'alors non démontrables, tant il est impossible, en 1885, de démontrer que les corps simples sont constitués d'une matière unique et fondamentale.

Jean Baptiste Joseph Delambre (1749-1822) donne également en 1817 le premier tome d'une Histoire de l'astronomie ancienne où il s'oppose aux « rêveries » de l'historien et astronome Jean Christophe Bailly qui avait fait la supposition d'un peuple perdu dont les connaissances perfectionnées auraient été transmises aux chinois et aux hindous. Pour contrer ces vagues hypothèses et remonter à la source exacte de ce que Delambre considère comme la véritable astronomie, c'est-à-dire l'astronomie mathématique, le savant indique qu'il lui a suffi de «remonter aux sources, et de consulter, dans leurs langues, les historiens et philosophes qui nous ont transmis ces notions vagues » (Delambre, $1817:$ v). Lui, con- 
sidère que ce qu'il nomme « science astronomique, c'est-à-dire une théorie qui lie tous ces faits mieux observés, qui en donne la mesure plus précise, qui fournit les moyens de calculer tous les phénomènes, qui sait en conclure les distances et les vitesses des corps célestes, leurs marches, leurs rencontres, leurs éclipses, et qui sait assigner les temps et la manière différente dont ces phénomènes s'offrirent aux habitants des divers pays » (Delambre, 1817 : v), considère que le fondateur de cette science ne saurait qu'être le grec Hipparque, relayé ensuite par Ptolémée. Il se propose alors de donner à ses lecteurs un aperçu des écrits des astronomes et mathématiciens grecs, en vérifiant et en réinterprétant si besoin est la mention des lieux et en traduisant le langage mathématique. L'enquête historique et philologique permet d'identifier précisément les dates et les lieux des observations décrites par Ptolémée ou Pline et de comprendre mieux ainsi leurs calculs, qui, en retour, permettent de vérifier mieux les lois déduites des observations des mouvements des astres plus contemporains (Ptolémée, 1813 : iv). La glose érudite, propre aux Belles Lettres, est mise au service de la démonstration de mesures et de calculs contemporains. Mais Delambre ne se contente pas de commenter les manuscrits antiques savants ; il consacre le septième chapitre de son ouvrage aux mentions de l'astronomie ou des astres dans les œuvres des poètes grecs et latins. Là le mathématicien commente et traduit les vers cités et indique à ceux qui pourraient par exemple considérer qu'Ovide était peu instruit en matière d'observation des astres qu'il n'a pas trouvé " une instruction plus réelle dans les derniers auteurs que nous avons été forcés d'extraire et que celles-ci du moins ne sont pas aussi sèches » (Ptolémée, $1813: 340$ ).

Si les astronomes cherchent dans l'Antiquité les documents à l'appui de leurs démonstrations mathématiques, les géologues qui se heurtent également aux temps profonds profitent des premiers progrès de la philologie comparée qui ne fera son entrée à l'Académie des Inscriptions et Belles Lettres que dans les années 1830 pour fournir des mythes et légendes une lecture littérale supposée prouver ou informer l'hypothèse d'un déluge universel. Dans le « Discours sur les révolutions à la surface du globe », en préface des Recherches sur les ossemens fossiles, $\mathrm{Cu}$ vier, en 1821, fait référence aux exégèses les plus récentes des textes de l'antiquité grecque et latine et aux découvertes et traductions sur les védas et pouranas hindous pour commenter ses textes et étayer sa théorie d'un Déluge (Cuvier, 1821 : LXXX-XC). Les preuves de la théorie géologique sont non seulement matérielles, mais aussi livresques; du moins l'interprétation de textes anciens en fait-elle des documents recelant un certain nombre d'informations susceptibles de faire preuve, à défaut de jamais pouvoir observer les premières révolutions du globe. Au passage, les catégories du mythe et de la légende sont repensées par les philologues et littérateurs qui en profitent pour modifier le corpus littéraire des œuvres dites canoniques.

C'est dire aussi que le régime de la preuve change et qu'à la déduction mathématique succède peu à peu l'idée de l'induction prégnante notamment dans les sciences du vivant. Mais il se pourrait aussi, du même coup, que le récit littéraire devienne une méthode d'argumentation, que la «littérature»vaille argument 
scientifique ou méthode savante, en tant qu'œuvre cette fois, et manière de composer un récit.

\section{L'usage heuristique}

L'usage heuristique du « littéraire » est le plus manifeste dans les discours savants qui entendent traiter des temps profonds ou des espaces infinis et qui ne peuvent recourir, au moins au tournant des XVIII ${ }^{\mathrm{e}}$ et $\mathrm{XIX}^{\mathrm{e}}$ siècles, à l'observation ou à l'expérimentation. Sous la plume d'un certain nombre de spécialistes des théories de la Terre, comme sous celle des naturalistes qui entendent mettre à jour des transformations ou des évolutions sur le long terme, le constat de l'impossibilité, pour l'esprit humain, de concevoir, de représenter et même de comprendre les phénomènes décrits est un véritable lieu commun. En d'autres termes, l'élaboration de critères propres de scientificité passe d'abord par la résolution d'un problème de poétique à laquelle peuvent concourir des œuvres ou des formes littéraires.

Dès 1809, Jean-Baptiste de Lamarck soulignait, au moment de défendre les variations des espèces dans la Philosophie zoologique, que la théorie de la fixité des espèces reposait sur une erreur de jugement : «L'apparence de stabilité des choses dans la nature sera toujours prise, par le vulgaire des hommes, pour la réalité ; parce qu'en général, on ne juge de tout que relativement à soi »; et « la brièveté de l'existence des individus de son espèce » est ce qui empêche l'homme de concevoir et d'appréhender les changements sur le long terme (Lamarck, 1809 : $71)$.

Mais les géologues, depuis Buffon jusqu'à Charles Lyell, répètent également le même constat et déplorent que les cadres de la représentation historique ne permettent guère de décrire un monde sans l'homme. Plus fondamentalement, au moment où Buffon, Cuvier, Buckland ou Lyell défendent l'idée que la géologie, grâce aux progrès accomplis en histoire naturelle, en chimie et en physique, sort de l'enfance de ses théories extravagantes et peut s'enorgueillir de certains faits, ils reconnaissent aussi la difficulté de démontrer par la seule observation ou par le raisonnement déductif leurs propres hypothèses. Les géologues ont de plus à lutter contre le cadre imposé du récit de la Genèse et le font en général en interprétant le texte biblique (Weber, 2019b : 104-116). Ils sont donc amenés le plus souvent à la fois à distinguer leur récit du mythe en en prônant une lecture allégorique, à condamner les fictions « romanesques » liées à la nécessité de respecter les cadres de ce récit et, dans le même temps à avoir recours à la fiction littéraire pour exposer au lecteur ce qu'il ne peut concevoir ou ce qui défie les règles $\mathrm{du}$ vraisemblable.

Certaines des fictions auxquelles ils ont recours se présentent, comme sous la plume de William Buckland lorsqu'il entend mesurer pour son lecteur l'étendue de la science géologique ou sous celle de Georges Cuvier à son lecteur la difficulté qu'il $\mathrm{y}$ a à statuer sur l'origine des espèces fossiles et l'impossibilité de l'homme fossile (Buckland, $1836: 1-3$; Cuvier, 1812 : 81-83), comme des hypothèses ou comme des expériences de pensée et relèvent des catégories du possible et de l'impossible. D'autres peuvent prendre la forme d'histoires contrefactuelles 
destinées à mettre en évidence les écueils de certains régimes de causalité lorsqu'ils sont érigés sur des présupposés fallacieux ; ainsi, Charles Lyell, dans Principles of Geology (1830: t. I, 87-88), explique les erreurs dans lesquelles sont selon lui tombés les tenants du «catastrophisme », en faisant imaginer à son lecteur les explications données par Champollion à la découverte des tombeaux égyptiens s'il avait été convaincu qu'aucune civilisation n'avait occupé les bords du Nil avant le $\mathrm{XIX}^{\mathrm{e}}$ siècle. Mais il arrive aussi que les savants recourent à des citations ou récritures d'œuvres littéraires en guise d'arguments ou d'hypothèses savants.

Lyell se sert notamment de The Rape of the Lock (1712) d'Alexander Pope, de Childe Harold (1812-1818) de Lord Byron et de Richard III (1591) de William Shakespeare. L'inscription de ces œuvres dans le tissu savant reflète différents degrés d'appropriation du texte littéraire par le texte savant. Les trois œuvres retenues ont cependant en commun d'engager un rapport particulier à l'Histoire : le poème héroïco-comique de Pope est un poème satirique qui se moque de la querelle entre deux familles d'aristocrates anglais aisément reconnaissables, Childe Harold's Pilgrimage est le porte-parole du désenchantement de toute une génération après les âges héroïques de la Révolution et des guerres napoléoniennes et Richard III clôt le cycle historique de la Guerre des Deux Roses. Ces trois textes littéraires illustrent la manière dont un texte littéraire peut être ancré dans un contexte historique particulier et valoir par-delà les bornes chronologiques de son intrigue. Lyell trouve ainsi dans la «littérature » des solutions poétiques et formelles pour décrire, dans une temporalité historique, des phénomènes ou des vérités qui excèdent le cadre temporel de la représentation des actions des hommes.

The Rape of the Lock est travesti en une récriture. Voulant démontrer que la situation de l'homme à la surface de la Terre a pu induire de fausses conclusions sur les âges de la Terre, Lyell imagine que le gnome Umbriel, depuis les souterrains où il vit, observe les couches géologiques; il en déduit que les couches les plus récentes sont celles du centre de la Terre où il vit et les couches les plus anciennes celles de la surface de la Terre :

But if we may be allowed so far to indulge the imagination, as to suppose a being entirely confined to the nether world some «dusky melancholy spirit », like Umbriel, who could «flit on sooty pinions to the central earth», but who was never permitted to « sully the fair face of light », and emerge into the regions of water and of air ; and if this being should busy himself in investigating the structure of the globe, he might frame theories the exact converse of those usually adopted by human philosophers (Lyell, $1830: 87-88)^{6}$.

\footnotetext{
6 «Mais laissons notre imagination aller plus loin encore, et supposons un être exclusivement confiné aux parties inférieures du globe, - un être à l'esprit sombre et mélancolique, qui, de même qu'Umbriel, ait ' 'le pouvoir de pénétrer, à l'aide de noires ailes, jusqu'au centre de la terre', mais à qui, en revanche, l'entrée des régions de l'eau et de l'air soit interdite, afin qu'il ne puisse, par sa présence, "souiller le pur éclat de la lumière". On comprend assurément que si un tel être se li-
} 
Lyell invente donc une expérience de pensée dont le héros parce qu'il est un personnage merveilleux peut entraîner le lecteur par delà les limites du possible et du vraisemblable. Le savant conclut son récit par un parallèle entre les thèses défendues par Leibniz et celles du gnome. Il donne droit de cité à la fiction dans le discours savant en en faisant le cadre d'une théorie savante au moins aussi exacte que celle des philosophes et le lieu de la dénonciation du caractère invraisemblable de leurs propos. Son récit incarne de plus la loi naturelle qu'il entend démontrer : la condamnation des théories de Leibniz n'est recevable que si l'on admet que ce qui y préside est du même ordre que ce qui présiderait aux théories du gnome ; aux mêmes causes, les mêmes effets.

Si The Rape of the Lock, par le biais de la récriture, se fond dans le discours savant, Childe Harold et Richard III sont simplement cités et commentés au titre de points de comparaison avec le discours des géologues. Le poème narratif de Byron survient au sein d'une argumentation destinée, dans le second tome, à démontrer la permanence des niveaux océaniques. Défendant l'idée de l'instabilité des terres, Lyell déplore que les observations faites sur l'engloutissement des baies de Baia et de la Conception n'aient pas entrainé la remise en cause de la théorie de leur stabilité. Le géologue digne de ce nom devrait dépasser les impressions naturelles qui, selon Lyell, ont poussé les poètes anciens à choisir le roc comme emblème de la stabilité et la mer comme métaphore de l'inconstance. Mais il devrait également suivre les intuitions qui ont mené les poètes modernes, et en particulier Byron, à voir au contraire dans les océans l'image de l'éternité. Là surgit la citation empruntée à Childe Harold: «- Their Decay / Has dried up realms to deserts : - not so thou / Unchangeable, save to thy wild wave's play: / Time writes no wrinkle on thine azure brow; Such as creation's dawn beheld, thou rollest now » (Lyell, 1830: t. II, 329). L'extrait, tiré de la strophe 182 du quatrième chant de Childe Harold's Pilgrimage conclut l'argumentation de Lyell comme il conclut presque, dans le poème narratif original, les méditations de Childe Harold sur l'instabilité des empires humains. Il invite les savants à imiter le poète dont les intuitions révèlent les lois de la Nature. Or, puisque les savants ont jusqu'alors cédé à des présupposés qui font de leurs théories des textes fictifs, il n'est nulle raison d'opposer la poésie narrative, nécessairement imaginaire et mensongère, au texte géologique. La littérature, sous la forme ici du poème narratif, fait office de prescience que les savants se doivent de démontrer ou d'invalider.

$\mathrm{Au}$ théâtre de Shakespeare, Lyell confère le soin d'invalider le jugement de ses pairs qui voudraient que textes littéraires et savants s'opposent par leur visée et par leur rapport à la vérité. L'œuvre dramatique est l'étalon à l'aulne de laquelle peut être jugée la vraisemblance de certains arguments savants. Lyell entend réfuter la théorie suivant laquelle l'examen des restes fossiles témoignerait d'un développement progressif de la vie animale et végétale, idée empruntée à

vrait à des recherches sur la structure du globe, les théories qu'il pourrait imaginer seraient complètement opposées à celles que conçoivent habituellement les observateurs placés à la surface de la terre » (Lyell, 1843 : 199-200). 
Humphrey Davy (Lyell, 1830 : t. I, 144-145). Il souligne que les probabilités de retrouver des carcasses de carnivores dans les couches anciennes recouvertes par les océans sont trop faibles pour qu'on puisse déduire une loi de leur absence. D'une part en effet, un cadavre animal a toutes les chances d'être dévoré par les requins ou autres poissons dont les dents ont été retrouvées dans les couches carbonifères et, d'autre part, si des restes d'animaux terrestres ont eu le bonheur d'atteindre la couche marine des sédiments, la transformation de ces sédiments en roches solides en a sans doute définitivement détruit les traces. Le rêve de Clarence, au premier acte de Richard III, est alors cité et commenté :

Clarence, in his dream, saw « in the slimy bottom of the deep », - a thousand fearful wrecks;

A thousand men, that fishes gnaw'd upon;

Wedges of gold, great anchors, heaps of pearl.

Had he also beheld amid "the dead bones that lay scatter' $d$ by", the carcasses of lions, deers, and the other wild tenants of the forest and the plain, the fiction would have been deemed unworthy of the genius of Shakespeare. So daring a disregard of probability, so avowed a violation of analogy, would have been condemned as impardonnable even where the poet was painting those uncongruous images which present themselves to a disturbed imagination during the visions of the night. But the cosmogonist is not amenable, even in his waking hours, to these laws of criticism; for he assumes either that order of nature was formerly distinct, or that the globe was in condition to which it can never again be reduced by changes which the existing law of nature can bring about. This assumption being one admitted, inexplicable anomalies and violations of analogy, instead of offending his judgment, give greater consistency to his reveries (Lyell, 1830: t. II, 149-150) ${ }^{7}$.

\footnotetext{
${ }^{7}$ « Clarence, dans son rêve, voit "sur le fond vaseux de l'Océan".

- A thousand fearful wrecks ;

A thousand men, that fishes gnaw'd upon;

Wedges of gold, great anchors, heaps of pearl.
}

Si parmi les "ossements humains dispersés sur le fond de la mer", Clarence eût aperçu aussi des squelettes de lions, de daims et de divers autres animaux sauvages qui habitent les plaines et les bois, dès lors la fiction eût paru indigne du génie de Shakespeare. Un si flagrant oubli des lois de la probabilité, une telle infraction aux règles de l'analogie eussent été impitoyablement condamnés, lors même que le poète ne s'en fût rendu coupable que pour peindre les images bizarres qui, pendant les visions de la nuit, se présentent à une imagination malade. [Mais le cosmogoniste n'est pas sensible, même dans ses heures d'éveil, à ces lois de la critique, car il suppose soit que cet ordre de la nature était autrefois distinct, soit que le globe était dans une condition à laquelle il ne pourra plus jamais être réduit par les changements que la loi existante de la nature peut apporter. Cette hypothèse étant admise, des anomalies et des violations d'analogie inexcusables, au lieu d'offenser son jugement, donnent plus de cohérence à ses rêveries] » (Lyell, 1843 : p. 370-371). Le passage que nous rajoutons entre crochets a été omis de la traduction française de 1846. 
La logique est celle de la démonstration par l'absurde. Les lois de la critique littéraire exigent qu'un récit, fût-il le récit imaginaire d'un rêve, soit jugé à l'aune du vraisemblable. Les géologues qui tirent de l'absence de restes fossiles des lois générales sur l'évolution des espèces ne tiennent pas compte des causes vraisemblables de cette absence. Car il serait invraisemblable de trouver des restes de daims et de lions au fond de l'océan. Il faudrait donc admettre que l'invraisemblable puisse être, en matière de géologie, un critère de vérité et de scientificité. Le «savoir» de la fiction est plus fiable que celui des savants et les lois de la critique littéraire peuvent révéler ou non le degré de vérité et de scientificité d'un récit savant. Les discours scientifiques de certains géologues sont des « rêveries » plus extravagantes que celles des poètes et ne doivent donc pas prétendre plus que ces dernières à une quelconque vérité. En une matière indécidable, comme l'est celle de l'évolution ou de la permanence des espèces, seule la vraisemblance peut trancher entre deux théories savantes. En l'absence de faits, de traces ou de documents, la démonstration scientifique procède moins par hypothético-déduction que par abduction.

Il faut donc aux savants géologues élaborer un régime de la preuve qui tienne de l'interprétation plausible, du possible, voire du vraisemblable plutôt que de l'observable. Les géologues trouvent l'exemple d'un tel raisonnement dans un texte littéraire ; le conte philosophique de Voltaire, intitulé Zadig ou la Destinée (1747), a en effet fourni à Georges Cuvier d'abord l'illustration parfaite de la méthode d'analyse des restes fossiles exposée en 1812 dans les Recherches sur les ossemens fossiles. L'ouvrage consacrait la naissance de la science de l'anatomie comparée. Georges Cuvier y indiquait que le principe de la corrélation des formes, loin de pouvoir être expliqué, reposait sur de multiples observations qui permettaient par exemple de savoir que la trace d'un pied fourchu révélait la présence d'un ruminant et concluait en ces termes : «c'est une marque plus sûre que toutes celles de Zadig » (Cuvier, 1834 : t. I, 184-185). En 1880, Thomas Huxley se référait à Cuvier pour ériger la «méthode de Zadig » en méthode scientifique devant s'appliquer à l'ensemble des disciplines relevant de l'histoire naturelle; le conte philosophique allait conférer au raisonnement par induction et par analogie, droit de cité dans les sciences. Pour ce faire, Huxley ouvrait l'essai « On the Method of Zadig. Retrospective Prophecy as a function of science » par une glose ironique du conte, feignant d'avoir échoué à découvrir la véracité historique de l'histoire de Zadig pour mieux affirmer que comptaient surtout les vérités proférées par le «philosophe » Zadig (Huxley, 1893 : t. IV, 1-3). Véracité historique et vérité savante ne se confondaient donc plus et le recours à la fiction imaginaire n'était plus incompatible avec la scientificité du discours savant.

Or dès 1870, l'historien Edgar Quinet, dans La Création, avait entrepris de « faire entrer la révolution contemporaine de l'histoire naturelle dans le domaine général de l'esprit humain » (Quinet, $1870:$ t. I,1) et désignait la géologie comme fer de lance de cette "révolution » méthodologique qui devait entraîner de nouvelles articulations entre sciences et arts. Si le langage en effet peinait à faire saisir les mondes «révélés d'hier», l'art pouvait contribuer à les représenter et l'imagination créatrice participer à l'élaboration des théories savantes : « Et pour- 
quoi les arts ne nous aideraient-ils pas à retrouver ce passé ? Si nous voulons faire rentrer dans les arts la grande imagination créatrice, n'est-ce pas là une voie qui s'ouvre d'elle-même et invite le génie à s'y engager ? ». Non seulement donc les artistes prendraient part aux progrès de la géologie, mais la géologie, en leur livrant de nouveaux sujets et de nouveaux thèmes, contribuerait à l'avènement d'un art nouveau, peignant les origines du monde. Quinet, pour qualifier cet art, n'inventait pas de catégorie nouvelle : «Si les artistes grecs et modernes étaient réduits à imaginer des alliances de formes impossibles, l'artiste dont je parle n'aurait, au contraire, qu'à puiser dans le monde organisé ; il aurait l'avantage de trouver sous sa main des formes toutes préparées dans l'atelier de la nature ; il pourrait ainsi être réaliste tout en dépassant les limites du monde actuel, ce qui semble le but suprême de l'art » (Quinet, 1870 : 35-36). La géologie ouvrirait aux écrivains et aux romanciers la voie d'un nouveau réalisme qui ne prétendrait ni à la conformité avec l'expérience vécue par le lecteur, ni à la véracité historique, mais à la vérité naturelle. Ainsi la définition de la «littérature », en tant que corpus d'œuvres souvent fictives ici, s'en trouverait renouvelée.

\section{Conclusion}

Les usages ornemental, documentaire et heuristique du « littéraire » dans le discours savant ne sont pas inventés au tournant des $\mathrm{XVIII}^{\mathrm{e}}$ et $\mathrm{XIX}^{\mathrm{e}}$ siècles. Mais, dans le contexte de la spécialisation des disciplines savantes et de l'émergence de la littérature en tant que corpus et discipline, ces usages deviennent problématiques et leurs conditions de possibilité sont en conséquence explicitées par ceux qui y ont recours. Ces conditions touchent à la fois à la redéfinition des méthodes scientifiques défendues et à l'élaboration d'acceptions nuancées de ce que les savants entendent par « littérature ». Sans doute n'est-il guère surprenant que les tenants de certaines disciplines savantes ne se concentrent que sur certains genres ou sur certains outils des études littéraires: que la géologie, comme l'Histoire, interroge la pertinence de l'usage de la fiction n'a rien de surprenant, que l'histoire naturelle, au moment de se distinguer de la philosophie naturelle, ait recours au conte philosophique est logique. Et les représentants des études littéraires auraient beau jeu de montrer que ce que les savants entendent par " littérature » n'est que la représentation qui convient le mieux à leurs démonstrations. Ce que montre l'étude de ces usages est qu'elle définit un spectre large de ce qui relève de la littérature, allant des répertoires de formes, de figures et de genres aux cuvres et aux méthodes d'analyses de la discipline. En d'autres termes, les savants qui font usage du littéraire, dès la fin du XVIII ${ }^{\mathrm{e}}$ siècle, loin de le réduire à un travestissement formel, explorent toutes les capacités heuristiques à la fois des œuvres et des méthodes de la discipline naissante (ne serait-ce que sous la forme de la philologie). Les trois usages, au sein d'un même texte, ne sont pas exclusifs l'un de l'autre.

On pourrait, suivant les remarques de Thomas Benatouïl déplorer que l'étude des usages du littéraire ne présuppose que la littérature puisse être usitée, et qu'on ne lui applique qu'une portée utilitaire (Benatouil, 2014 : 31). Or la litté- 
rature est précisément née du refus de la rhétorique. Il apparaît cependant que l'estimation politique contemporaine des « retombées » des études littéraires en termes de compétences a occasionné un retour en force de la réduction de la discipline littéraire à l'apprentissage d'un art d'écrire, voire, plus rarement d'argumenter. Or, l'étude littéraire des textes savants qui empruntent à la sphère de la « littérature » montre fort bien que les savants eux-mêmes reconnaissent à la littérature un autre usage que celui de la rhétorique et consacrent d'une certaine manière l'efficacité scientifique des facultés heuristiques auxquelles la lecture et les analyses littéraires des œuvres prédisposent leurs tenants. Ceux-ci pourraient s'en souvenir parfois lorsqu'il s'agit d'évoquer l'utilité, voire la nécessité des études littéraires.

\section{RÉFÉRENCES BIBLIOGRAPHIQUES}

BARRÈRE, Anne \& Danillo MARTUCELli (2009): Le Roman comme laboratoire : de la connaissance littéraire à l'imagination sociologique. Villeneuve d'Asq, Presses Universitaires du Septentrion.

BenatouïL Thomas (2014) : «J'écris pour des utilisateurs », in Hervé Oulc'hen (dir.), Usages de Foucault. Paris, Presses Universitaires de France, 31-42.

BERnARDIN DE SAINT-PIERRE, Jacques Henri (1984 [1788]): Paul et Virginie. Paris, Gallimard (« Folio classique »).

BeRTHELOT, Marcelin (1885) : Les Origines de l'alchimie. Paris, Georges Steinheil.

BRosseau, Marc et Micheline CAMBron (2003) : « Entre géographie et littérature : frontières et perspectives dialogiques », Recherches sociographiques, 44:3, 525-547. URL : https://id.erudit.org/iderudit/008205ar.

BUCKLAND, William (1837): Treatise on Geology. London, Pickering, t. I.

BUFFON, Georges Louis Leclerc (1827) : Euvres complètes de Buffon. Paris, Delangle, t. I.

CASTRES, Antoine Sabatier de (1770) : Dictionnaire de littérature. Paris, Vincent, t. III.

CERTEAU, Michel de (1980) : L'Invention du quotidien. Paris, Gallimard.

CHASsot, Fabrice (2011) : Le Dialogue scientifique au XVIII siècle. Paris, Garnier.

CUVIER, Georges (1821): «Discours préliminaire», Recherches sur les ossemens fossiles. Paris, Dufour et D'Ocagne, t. I, I-XC.

CUVIER, Georges (1861a) : «Éloge historique de Daubenton », Recueil des éloges historiques. Firmin Didot Frères, 3-36.

CUVIER, Georges (1861b) : «Éloge historique de Lacépède », Recueil des éloges historiques. Firmin Didot Frères, 371-409.

DelAmBRE, Jean Baptiste Joseph (1817) : Histoire de l'astronomie ancienne. Paris, $\mathrm{V}^{\mathrm{ve}}$ Courcier, t. I.

DIDEROT, Denis \& Jean-Baptiste Le Rond D’AlEMBERT (1786) : Encyclopédie. Paris, Brisson, t. 15. 
GeOFFroy SAINT-HILAIRE, Étienne (1830) : Principes de philosophie zoologique discutés en mars 1830 au sein de l'Académie royale des sciences. Paris, Pichon et Didier.

Horace (1821) : Ars poetica, Euvres poétiques d'Horace. Campenon (éd.), Paris, Boucher, t. II.

HUXLEY,Thomas (1893) : «On the method of Zadig. Retrospective Prophecy as a function of Science », in Science Hebrew Tradition, Collected Esays. London, Macmillan, t. IV, 1-37.

JABLONKA, Ivan (2014) : L'Histoire est une littérature contemporaine. Paris, Seuil.

JABLONKA, Ivan (2016) : Laëtitia ou la fin des hommes. Paris, Seuil.

JULLIEN, Bernard (1866) : Traité des figures et des formes du style. Paris, Hachette.

LAMARCK, Jean-Baptiste de (1809) : Philosophie zoologique. Paris, Dentu.

LYELL, Charles (1830) : Principles of Geology, London, Murray.

LYELL, Charles (1843) : Principes de géologie. Traduction française de Tullia Meulien, Paris, Langlois et Leclercq, t. I et t. II.

MARChal, Hugues [dir.] (2013): Muses et Ptérodactyles. La poésie de la science de Chénier à Rimbaud, Paris. Éditions du Seuil.

NORDEAU, Max (1892) : Entartung, Berlin, Carl Duncker Verlag.

NordeAu, Max (1894) : Dégénérescence. Traduction française d'Auguste Dietrich. Paris, Félix Alcan.

PIETTE, Albert (2017) : Le volume humain. Esquisse d'une science de l'homme. Paris, Les Bords de l'eau.

PlayfaIR, James [éd.] (1822) : «Biographical Memoir », The Works of John Playfair, Edinburgh, Archibald Constable and co, t. 1.

PlAYFAIR, John (1812) : «Elements of Geometry, Geometrical Analysis and Plane Trigonometry [...] by John Leslie », The Endinburgh Review, 20.

PtolÉmÉe (1813) : Composition mathématique. Traduction de P. Halma. Paris, Henri Grand, t. I.

QUINET, Edgar (1870) : La Création. Paris, Librairie internationale, t. I.

WANLIN, Nicolas [dir.] (2019) : Littérature et sciences au XIX ${ }^{e}$ siècle. Une anthologie. Paris, Garnier.

WEBER, Anne-Gaëlle (2016) : «Alexander von Humboldt: un précurseur de l'écopoétique ? », Loxias, 52. URL : http://revel.unice.fr/loxias/index.html?id-8289.

WEBER, Anne-Gaëlle (2019a): «Goethe et les sciences : une histoire de métamorphose », in Fabula / Les colloques. Goethe, le mythe et la science. Regards croisés dans les littératures européennes, URL: http://www.fabula.org/colloques/document6101.php.

WEBER, Anne-Gaëlle [dir.] (2019b) : Passerelles, entre sciences et littératures. Paris, Garnier. 